\title{
Inexpensive Database Management Software
}

\author{
Carl Grafton and Anne Permaloff, Auburn University at \\ Montgomery
}

In our last $P S$ article, we discussed the varieties of data that political scientists store, retrieve, and analyze using microcomputers. ${ }^{1}$ The best software tools to use depend on the kinds and amounts of data.

For medium to large quantities of numerical data, the best tool for input and retrieval is a traditional database management program such as dBASE or Paradox. A large data set is defined as one that by itself or together with related transformation and query needs exceeds a computer's RAM capacity. Traditional database management programs may also be best for text-based entries if each entry is approximately 250 characters or less in length. They may also be the tools of choice for both numerical and text-based entries (again of 250 characters or less) if complex query operations are conducted only infrequently.

\section{Major Components of Database Management Programs}

Although database management programs differ in appearance and capabilities, most consist of a few basic components: database structure definition, data input, tabular display, search, sorting and indexing, selective listing, and reporting.

The database structure definition component allows (and requires) the user to inform the program of the names of fields (variables in a statistics program and columns in a spreadsheet), their length, and their type. For example, a database containing state legislators' last names, first names, and district numbers could have field names such as LAST_NAME, FIRST_NAME, and DISTRICT. Their length would be specified as the greatest number of spaces that will be taken up by the longest element in each field, perhaps 15,10 , and 2 , respectively. Field types may be character, numeric, date, logical, or memo. Character fields are for text or numbers such as telephone numbers that will not be used for mathematical calculations. Numeric fields are for numbers and date fields for dates. Logical fields allow entries specifying Yes/No or True/False conditions. Memo fields permit long (often multi-screen) text entries, but the contents are not subject to searches.

A database management data input module lists field names on a blank screen and allows data to be keyed in one record (set of fields) at a time. A good data input module allows for free movement between fields and records. Furthermore, when data are being keyed in, it is important that movement from field to field and from one completed record to the next empty record be accomplished entirely with the ENTER key. Some programs require that the user press a function key or some other key when a record is complete. Such movement wastes time and invites mistakes.

Most database management programs allow data to be displayed and edited in a tabular display that looks like a spreadsheet. Each column is a field and each row a record.

Database management searching and listing subsystems allow record characteristics to be specified by Boolean logic statements. Searches locate records one at a time while lists display all specified records at once.

Records can be sorted by alphabetical or numeric order. Typically, this process results in the creation and saving of a new file in the specified order. For a large database, sorting is time consuming, and the new file absorbs as much disk space as the original file. Most database management programs offer the attractive alternative of indexing which creates the functional equivalent of a book index. Indexing is faster and uses less disk space than sorting. As with sorting, a file can be indexed by any field or sometimes combination of fields. For example, a database of legislators can be indexed by their last names. When the file is listed, the names appear in alphabetical order even though a sort has not been performed.

Database management report modules allow data to be printed in formats defined by the user. Report formatting options typically include page titles, the display of only selected fields, the use of a particular index, column headings, and subtitles. A well designed report can make data much more readable than a simple list.

\section{Relational Database Management Capabilities}

The term relational is commonly applied to database management programs, but it is used in two ways. One refers to Boolean logic applied to searching and listing, virtually a universal feature of database management programs. The other refers to connections that can be established between or among files by linking them according to common fields or columns. Such a relational database management program can be extremely useful to a political scientist. An example will show why, and explain the basic relational concept.

State legislators are frequently listed in official records by name, district number, and county. These data are easily entered in many statistics packages, all spreadsheets, and all database management programs in terms of the column or field headings shown in Figure 1. Legislative committee memberships are usually listed by committee and legislators' last names, also illustrated in Figure 1. However, if an analyst is interested in committee memberships in terms of which counties are represented, a bridge must be built between the two data structures, repre- 
FIGURE 1.

Two Database Files Linked by Common Field "LAST_NAME"

Legislator Database

\begin{tabular}{lll}
\hline LAST_NAME & FIRST_NAME & COUNTY \\
\hline 1 & & \\
\hline 2 & & \\
\hline 3 & & \\
\hline$\cdot$ & Committee Database \\
\hline$\frac{1}{\text { COMMITTEE }}$ \\
\hline$\frac{2}{3}$ \\
\hline
\end{tabular}

List Based on Relationship Between Databases

\begin{tabular}{lll}
\hline LAST_NAME & COMMITTEE & COUNTY \\
\hline 1 & \\
\hline 2 & \\
\hline 3 & \\
\hline$\cdot$ & \\
\hline
\end{tabular}

sented here as two files. In this case the bridge is based on the LAST NAME field found in both files. A relational database management program can support a query that asks, for example, how many representatives from Montgomery County may be found on the Finance Committee. Relational capabilities are not always needed, but they can be very helpful in some circumstances. Database management programs lacking relational capabilities are usually referred to as flat file programs.

\section{The Cost of Database Management Software}

Commercial database management programs vary in price from $\$ 45$ for Clarion Personal Developer to $\$ 498$ for the highly regarded Paradox (both prices mail order discount). Except for Clarion, no standard commercial relational program is available for less than about $\$ 180$, and most cost considerably more.

A number of powerful and easy to use shareware database management programs rival their standard com- mercial counterparts and even exceed them in a few respects. Making these products even more attractive is the fact that shareware versions are available for $\$ 3.00-\$ 4.00$ from shareware distributors. This allows experimentation before purchasing the program one will ultimately use.

Regular use of a shareware program ethically obligates the user to buy the program from the developer. The purchaser usually benefits by receiving both a full printed manual rather than the typical shareware manualon-disk and a newer and more powerful program than the one distributed through shareware channels. The cost of such programs is usually far less than their commercial counterparts.

We will examine several shareware programs and the inexpensive Clarion Personal Developer. Our objective is to narrow a cost conscious user's search to one or two programs.

\section{Hardware}

All of the programs discussed here operate on IBM or IBM-compatible computers. A survey of public domain and shareware distributors yielded no inexpensive Macintosh database management programs.

The programs examined need a hard disk for effective use, and the better ones require 640K RAM.

\section{Features}

The examined programs are menu driven, although some allow the user to create full applications in a largely dBASE compatible language. An application is a custom tailored database management program designed for a specific use such as population analysis by precinct or legislative support scores for a president's programs. Our focus will be on menu operations because we believe that the typical political scientist will be using these tools for analysis rather than creating applications except perhaps for input screens that might be used by computer novices for such purposes as recording telephone poll results.

The programs will be rated (see Table 1) according to the following list of desirable features: 
TABLE 1.

Relational Database Management Program Performance Characteristics

\begin{tabular}{lccccc}
\hline Feature & Clarion & $\begin{array}{c}\text { Data }+ \\
\text { Plus }\end{array}$ & 3!! & Wampum & Zephyr \\
\hline dBASE file compatibility & 5 & 5 & 5 & 5 & 5 \\
dBASE menu compatibility & 0 & 3 & 4 & 2 & 2 \\
General ease of use & 3 & 3 & 5 & 3 & 3 \\
Prompts and help & 3 & 2 & 5 & 2 & 2 \\
Relational & 5 & 4 & 5 & 3 & 3 \\
Modifiable database & 4 & 5 & 5 & 2 & 5 \\
Input screen paint & 4 & 5 & 0 & 0 & 0 \\
Report & 4 & 4 & 5 & 4 & 4 \\
Path set & 5 & 4 & 5 & 0 & 0 \\
Infrequent disk drive & 5 & 4 & 0 & 4 & 5 \\
Import/Export capability & 4 & 5 & 0 & 5 & 5 \\
Macro & 0 & 0 & 5 & 5 & 5 \\
External DOS & 5 & 5 & 5 & 5 & 5 \\
\hline
\end{tabular}

\section{Flat File Performance Characteristics}

\begin{tabular}{lccccc}
\hline Feature & $\begin{array}{c}\text { File } \\
\text { Express }\end{array}$ & PC-File & $\begin{array}{c}\text { File } \\
\text { Base }\end{array}$ & $\begin{array}{c}\text { Carte } \\
\text { Blanche }\end{array}$ & Wynfield \\
\hline dBASE file compatibility & 0 & 5 & 0 & 5 & 0 \\
dBASE menu compatibility & 0 & 0 & 0 & 0 & 0 \\
General ease of use & 5 & 5 & 3 & 4 & 4 \\
Prompts and help & 4 & 5 & 3 & 3 & 4 \\
Modifiable database & 2 & 5 & 1 & 5 & 5 \\
Input screen paint & 5 & 5 & 0 & 0 & 5 \\
Report & 4 & 5 & 5 & 0 & 2 \\
Path set & 5 & 5 & 0 & 5 & 5 \\
Infrequent disk drive & 5 & 5 & 5 & 5 & 5 \\
Import/Export capability & 4 & 5 & 0 & 0 & 2 \\
Macro & 0 & 0 & 0 & 0 & 0 \\
External DOS & 0 & 5 & 5 & 0 & 5 \\
\hline \multicolumn{1}{c}{$0=$ Nonexistent or very poor } & $3=$ Adequate & $5=$ Excellent &
\end{tabular}

- dBASE file compatibility,

- dBASE menu compatibility,

- general ease of use,

- prompts and help screens,

- relational capabilities,

- modifiable database format,

- input screen painting,

- report capabilities (formatted output),

- path flexibility for data files,

- infrequent disk drive access,

- import/export capability,

- macro capabilities,

- ability to access DOS from within the program.

Some of these points require explanation. dBASE compatibility is important because, despite the growth of dBASE's competitors such as Paradox and R:Base, dBASE is still the major standard in database management programs if dBASEoriented programs such as Clipper and Foxbase are counted.

Path flexibility for data files means that data files may be placed on any drive and directory and not just the drive and directory on which the database management program is located.

Modifiable database format refers to additions, deletions, and movement of fields. The equivalent in a spreadsheet is column insertion, deletion, and movement. This is an important feature because no matter how carefully a database structure is planned, it is likely that changes will be desired after large amounts of data have been entered. Again, the working assumption behind this article is that database management programs are most likely to be used by political scientists for analysis; and that analysis may move in unpredictable directions.

Input screen painting is a feature found on virtually all standard commercial database management programs. It allows the user to design a data input screen using a series of menu choices and pointing with a cursor.
Import/export capability refers to the variety of file formats that a program can read. Ideally, these should include space or comma delimited ASCII, dBASE, and DIF.

\section{Relational Database Management Programs Examined}

All of the relational programs are dBASE file compatible except for Clarion Personal Developer. Clarion can, however, import and write files in the dBASE format.

The products discussed below are listed in alphabetical order.

Clarion Personal Developer, version 2.0. Clarion Software Corporation, 150 East Sample Road, Pompano Beach, FL 33064. (305) 7854555. $\$ 45$ (mail order discount).

Data + Plus, version 3.98. Plano Computer Sales Co., 1001 Lockhart, Plano, TX 75023. (214) 517-8662.

Full version (copy protected) without printed manual but with one year telephone support $\$ 39.95$ plus $\$ 5.00$ shipping and handling. Shareware version from Gemini Marketing, Inc., P.O. Box 640, Duvall, WA 98109-0640. (800) 346-0139, (206) 788-4295. FAX (206) 788-0717. All Gemini disks are $\$ 3.00$ plus $\$ 4.00$ shipping and handling per order.

1 on $I=3$ !!, version 1.5 .1 on 1 $=3 ! !, 26$ Finchwood Dr., Trumbull, CT 06611. (203) 375-0914. Newest version with printed manual $\$ 69.00$. Shareware version from Computer Solutions, P.O. Box 354, Mason, MI 48854. (800) 874-9375 or (517) 6282943. All Computer Solutions disks are $\$ 3.49$ plus $\$ 4.00$ shipping and handling per order.

Wampum, version 4.05. Ward Mundy Software, 4160 Club Drive, Atlanta, GA 30319. No telephone. Newest version with documentation on disk $\$ 25.00$ plus $\$ 5.00$ handling; with printed, bound documentation $\$ 45.00$ plus $\$ 5.00$ handling. Shareware version from Computer Solutions (see above).

Zephyr, version 1.0. Ward Mundy Software (see above). Newest version with printed, bound documentation $\$ 65$ plus $\$ 5.00$. Shareware version from Computer Solutions (see above). 


\section{Clarion Personal Developer}

Clarion Personal Developer is the best product discussed in this article for the relatively easy development of complex specialized applications. Custom data input screens, tabular displays, complex relational reports, help screens, and other features can be designed by this menu-driven program without the need for difficult programming that would be required with the other programs represented here that are capable of generating applications. The process of application development is aided by a well written manual, adequate help screens (called up by pressing F1), but very skimpy prompts (aides that appear on screen routinely). A basic, flat file application can be developed in a few minutes; a relational application takes approximately one hour.

Clarion-generated applications have a highly professional look and feel. Menu choices are implemented with windows and bar-cursors. The standard screens are attractive. Application modules connect flawlessly. Clarion would be our first choice for a product to be delivered to a client who needed a personnel database system or a telephone poll data input program.

Unfortunately, Clarion's superb application development capabilities come at a high cost in terms of the analytical flexibility that most political scientists require. Once data input screens, report formats, and interfile relationships have been established, changing them is a time consuming and awkward process.

Clarion uses its own file format, but it can read and write dBASE II and III, DIF, and comma delimited formats. Importing or exporting files in these formats can be made part of an application developed with Clarion.

\section{Data + Plus}

This is an extraordinarily impressive program. At first glance, its logically structured menu system is not much like the one employed by dBASE, but a little familiarity with dBASE will be all that is generally required for easy use of Data + Plus.

The program's strong points are many. It is the only program other than Clarion that permits the easy creation of menu-driven applications. An existing database or application can be easily modified by adding or deleting fields (unlike a Clarion application). It is ithe only relational program evaluated here except Clarion that has an input screen painting capability. It can import and export space delimited ASCII files, and external DOS commands can be executed from within the program.

The shareware version of Data + Plus lacks relational capabilities and application development features including data input screen painting. The full version purchased from the manufacturer is copy protected. This copy protection permits two installations, and an uninstall program allows Data + Plus to be shifted to another machine.

Data + Plus's major operational weakness is that its prompt and help screens and documentation are not as complete, clear, and detailed as they should be. For example, an important movement from one part of the program to another is performed by pressing the PgDn and PgUp keys, but this critical piece of information does not appear as a prompt or as part of a help screen nor was it located in a search of the documentation. Data + Plus's relational capabilities are also poorly documented, and screen information on this critical topic is virtually nonexistent.

\section{1 on $1=3 !$ !}

This oddly named program rivals Data + Plus in overall quality although the two have different strengths and weaknesses. 3!! offers the most dBASE-like menu system of any of these programs. A book on dBASE III Plus can serve as a 3!! manual.

3 !! also offers superb prompt and help screens. Detailed prompts are always present, and help screens provide context sensitive assistance in even greater depth. This makes 3!! an excellent choice for the occasional user unless the program's few weaknesses present a serious problem.

3 !! allows the user to select any disk drive and any directory for data. It permits complete flexibility in field deletion or addition. Its relational capabilities are considerably easier to use than the other programs (except Clarion) or dBASE III Plus itself, and it supports macro creation. Its report generation module is powerful and easy to use.

The program's biggest weakness is that it is extremely disk intensive; every menu choice results in disk drive access. This problem can be virtually eliminated by using a disk cache program such as the one provided in PC Tools Deluxe. Since 3!! also requires a full $640 \mathrm{~K}$ RM, the cache must reside in expanded or extended memory. 3!! lacks both an input screen painting capability and custom input screen creation through programming.

\section{Wampum}

Wampum is not as functional for the infrequent user as Data + Plus or $3 ! !$ because its prompts and help screens do not provide nearly as much assistance, although its printed manual is excellent. Wampum's menus are not similar to dBASE's. Its data files must be on the same drive than it is on. It has no input screen painting feature although custom input screens can be created via programming. File structure modification is limited and awkward, and relational capabilities are not as easy to use as they could be.

Far too many routine tasks are difficult to accomplish with Wampum. For example, in the spreadsheet-like browse mode, adding data for a newly added field requires too many steps.

Despite its weaknesses, Wampum is an excellent value. Its major problem is that it suffers by comparison with better programs.

\section{Zephyr}

Zephyr is produced by the same manufacturer as Wampum. Although Zephyr is the newer program, it shares Wampum's poor prompt and help screen features as well as its fine documentation. Zephyr is better than Wampum in other ways, but as Table 1 indicates, it is still inferior in most respects to Data + Plus and $3 ! !$. 


\section{Flat File Programs}

The following flat file programs will be covered:

Carte Blanche, version 4.01. Functional Software, $920 \mathrm{~N}$. Washington, Edmond, OK 73034. (405) 340-1940. Newest version, printed manual, one year telephone support $\$ 60.00$. Shareware version from Gemini (see above).

Filebase, version 7.95. EWDP Software, Inc., P.O. Box 40283, Indianapolis, IN 46240. No telephone number available. Newest version with printed manual $\$ 30$. Newest version without manual $\$ 15$. Shareware version from Gemini (see above).

File Express, version 4.32.

Expressware Corp., P.O. Box 230, Redmond, WA 98073. (206) 788-

0932. Without printed manual $\$ 15.00$ plus $\$ 5.00$ shipping and handling. Newest version (5.0) with printed manual $\$ 99.00$ plus $\$ 5.00$ shipping and handling. Shareware version (4.28) from Gemini (see above).

PC Data Controller, version 3.8. Richware, 50 Meredith Rd., Tewksbury, MA 01876. (508) 851-4554. Newest version with on-disk manual $\$ 29.95$. Shareware version from Gemini (see above)

PC-File, version 5.0. ButtonWare, P.O. Box 96058, Bellevue, WA 98009. (800) 528-8866. Newest version and manual $\$ 134.95$. Shareware version from Gemini (see above).

Wyndfields, version 1.1. Wyndware, 101 E. Milwaukee St., Suite 423, Janesville, WI 53545. (608)

755-1628. Newest version and manual \$70. Shareware version from Gemini (see above).

In discussing flat file packages, we will depart from our usual practice of examining programs individually. Even though several of them are fine products, all are markedly less useful than Data + Plus, 1 on $1=3 !$ !, and Clarion, which are less expensive than several flat file programs.

Flat file programs are widely regarded as being easier to use than relational programs, but none of the flat file programs are as easy to use as Data + and 3!!. File Express and the elegant PC-File come the closest, but neither is as efficient a tool for data entry as any of the relational programs. With the relational pro- grams, the entire data entry process including record completion (writing the record to a RAM buffer or directly to disk) is operated with the enter or return key. File Express and PC-File require that the F10 key be pressed during data entry.

Distractions are common in data entry, and it is frequently necessary to examine the last complete record. All of the relational programs allow the last record to be viewed by pressing a single key, usually PgUp. Neither File Express nor PC-File allows an easy return to the last complete record during data entry.

Of all the flat file programs, only PC-File has characteristics that are in any way superior to the relational programs. PC-File has a data input screen painting module that is easier to use than those contained in Data + Plus and Clarion. PC-File is unique among the flat and relational programs examined in its graphing capabilities. These include pie charts, bar charts, and scatter plots. PC-File also allows the importing and exporting of more file formats (dBASE, DIF, fixed length, comma-delimited, space delimited, and WordPerfect) than any program in either category.

\section{Conclusions}

Unless one of PC-File's few unique features is important, deciding among the several high quality programs represented here is easy. 1 on $1=3 !$ ! is the best choice for the occasional user whose needs are primarily analytical. Data + Plus is better if custom input screens are required, if relatively simple applications are being developed, or if disk caching is not available. Clarion Personal Developer allows the relatively easy creation of highly complex applications, but it is too rigid a tool for convenient or frequent experimental query operations.

\section{Note}

1. Anne Permaloff and Carl Grafton. 1990. "Text Oriented Database Management," PS: Political Science \& Politics 23 (December): 586-91.

\section{Quick Notes}

Presidential Campaign!, Version 1.1, by G. David Garson. National Collegiate Software, Duke University Press, 6697 College Station, Durham, NC 27708. (919) 684-6837. \$59.95 ( $\$ 47.95$ for educators). IBM or compatible, 384K free RAM, one 3.5" disk drive (hard drive recommended for keeping student records).

In this recently released simulation the player runs for president in a highly simplified but instructive context against a computer incumbent or challenger of either party. The player controls financial resources that can be allocated among states or regions for advertising. The player may also choose to buy polling information or invest in fund-raising activities. The polling data may be printed. In addition, the player must respond to situations that arise during the campaign such as the opponent's challenge to debate or the possibility that her or his campaign manager has cheated on income taxes.

The instructor may add situations and determine the impact of player responses on the outcome. This can be accomplished with virtually any word processor. The complete text of situations provided in the simulation is included in the manual and covers 65 pages.

Once the player has become acclimated to the simulation, it takes less than an hour to complete. A simulation may be saved in progress and restarted another time.

The instructor may configure the simulation to ask the player the reasons for his or her decisions. These explanations may take up to 24 lines of text. Records of player performance may be stored for later evaluation by the instructor. The simulation may also be configured to allow players to print the contents of decisions together with feedback from the computer and bibliographic citations. If the instructor feels that students would show these materials to others before they run the simulation, this feature can be disabled.

The Presidential Campaign! manual suggests that the program can be used for homework, controlled experimentation, or in class with a projection system. We agree 
that it would be appropriate for these applications.

Presidential Campaign! is a smooth running and professional looking program that would make an excellent addition to a course on political behavior, the presidency, or campaigning.

\title{
Can American Social Science Majors Acquire an International Perspective?
}

\author{
Remi Clignet, University of Maryland, College Park
}

Concern is growing over the lack of sensitivity that American social sciences students display toward the rest of the world (Brademas 1983; Tiryakyan 1986; Shenon 1989; Hechinger 1989). In economic terms, this insensitivity is seen as preventing free trade; in political terms, as hampering effective diplomacy. In scientific terms, it prevents a proper use of comparative methods.

Since the curriculum represents an "ideological superstructure," innovations will alter students' world views only if they have been preceded by changes in the organization of academic institutions and disciplines. The effectiveness of curricular innovations requires a tightening of the links among disciplines and universities. Further, the effectiveness of these innovations depends also on a dialectic resolution of the tensions between the purity of disciplines and their relevance to the abilities and aspirations of students. Because students initate the beliefs and behaviors of their familial, academic, and disciplinary environments, these three environments combined should determine the range of the reforms proposed (Bourdieu et Passeron 1964, 1970). ${ }^{1}$

\section{Provincialism of the Culture At Large}

"Most of the students at an overwhelming majority of institutions of higher learning are simply going through their careers without exposure to any non-American experience" (Harf 1970). As this lack of exposure reflects the insensitivity of many American parents to international events, its origin is manifold. It results in part from the latent isolationism of American culture. When Americans describe themselves as a nation of "immigrants in time," they emphasize the need to renew constantly the ideological justification of their lives. Their search for self-identity combines the rejection of the past and the dream of a future held synonymous with continuous progress (Bellah et al. 1984).

\section{"Most of the students at an overwhelming majority of institutions of higher learning are simply going through their careers without exposure to any non-American experience",}

In contrast to Europeans who value highly the "moss" of the past and its traditions, Americans hold that it is a good thing that "rolling stones gather no moss." But this blindness to time and history usually dulls sensitivity to other voices and other shores. ${ }^{2}$ Despite the relentless efforts of some Americans to promote international cooperation and to enhance the status of the United Nations or of UNICEF, many of their countryfolks are attracted by isolationism. ${ }^{3}$ Whether the economic and political dominance of the United States in the world is asserted by Americans themselves or acknowledged by the international community, it lowers individual sensitivity to other cultural and political entities. Indeed, Pax Americana does not pre- dispose American adults or students to acquire a critical knowledge of other viewpoints or cultures.

The immensity of the country and the attraction exerted by a mythical West have helped Americans to focus their attention inward rather than outward. In contrast to Gemeinschaft-like cultures where personal identity evolves as a result of social transactions with local significant others, American mythology and the notion of the "imperial Self" emphasize the role played by mobility in the definition of the Self (Anderson 1971). Since mobility entitles individuals to "try everything at least once" (Bellah et al. 1984) or, "if something does not work here, to try it elsewhere," it generates a superficial mode of social interaction that is rarely challenged.

To illustrate, Americans had no difficulty singling out high school chemistry or physics programs as the major cause of the country's inability to immediately match the success of the Soviet Sputnik. In contrast, they have been more reluctant to appreciate the consequences imputed to their lack of international sensitivity and, for example, to appreciate "what was and is in the mind of the Iranian population" (Hechinger 1987). "The point is that this environment teaches students to be indifferent to whatever fails to stir the enthusiasm of their teachers and parents.

\section{Provincialism Fostered by Academic Decentralization}

Two major factors tend to impede or prevent any improvement in the teaching of international skills. First, the independence of colleges and uni- 